\title{
CONTRIBUCIÓN DE LOS PRINCIPIOS COSERIANOS A LOS ESTUDIOS CONTRASTIVOS
}

\author{
OLGA MORI \\ Universidad de Münster
}

0 . No cabe duda del valor de la teoría de Eugenio Coseriu para los estudios contrastivos. En efecto, toda su teoría se basa en la observación del funcionamiento de las unidades de una lengua en los distintos niveles de estructuración lingüística y en la comparación de las mismas con las de otras lenguas. Es cierto que, al realizar estudios contrastivos siguiendo su teoría estructural funcional, es necesario, en primer lugar, conocer los fundamentos de la misma. Sin embargo, aquí haremos hincapié sólo en algunos de sus principales trabajos de enfoque contrastivo.

Cometido esencial de Coseriu en toda su obra es distinguir entre planteamientos correctos y falsos, hacer nuevas distinciones y delimitar claramente los campos de cada rama de la lingüística y su objeto de estudio, propósito que también es evidente en el caso de los estudios contrastivos.

1. A comienzos de los años setenta todavía reinaba inseguridad acerca de la designación correcta de la materia cuyo objeto de estudio es la comparación sincrónica de las lenguas. Debido a ello, los estudiosos se planteaban numerosas preguntas: ¿qué designaba propiamente el término lingüística contrastiva? ¿por qué alternaba con gramática contrastiva y con gramática confrontativa? Tampoco se había delimitado con exactitud el campo que abarcaban las dos últimas ni su finalidad: ¿qué estudiaban realmente? ¿era la gramática contrastiva la lingüística que consideraba sólo las diferencias y era aplicada a la enseñanza de las lenguas extranjeras? ¿cómo se relacionaba ésta con la traducción, la descripción de las lenguas, la psicología lingüística y los estudios dialectales?

La designación gramática contrastiva proviene del inglés contrastive grammar y la de gramática confrontativa se le adjudica a L. Zabrocki (1970, 33), germanista de Leipzig, según el cual, la primera se ocupa sólo de las diferencias entre las lenguas y la segunda trata tanto las semejanzas como las diferencias. A pesar de que, con los resultados obtenidos de los estudios contrastivos, se espera lograr mejores resultados en la enseñanza de las lenguas, el hecho de que la gramática contrastiva sólo tenga en cuenta las diferencias no

Odisea, $\mathrm{n}^{\circ} 3,2003$, ISSN $1578-3820$, pags. $167-178$ 
siempre tiene resultados positivos en la enseñanza porque existen otros factores causantes de errores (Di Pietro 1972).

En un simposio dedicado especialmente a la gramática contrastiva, Coseriu (1970a, 175177) resume y evalúa los resultados de las exposiciones presentadas y se empeña en delimitar las ciencias que comparan las lenguas y sus campos de estudio. Sugiere, entonces, distinguir dos tipos de gramática y llamar gramática contrastiva a aquella que confronta «textos» de distintas lenguas con fines prácticos, principalmente en la enseñanza de lenguas extranjeras, es decir que se ocupa del plano de la designación. La gramática confrontativa estaría dirigida hacia la comparación de las lenguas con la meta de obtener resultados científicos de la confrontación de ámbitos relacionados de diferentes lenguas. Esta ciencia estudiaría tanto las analogías como las diferencias de la construcción lingüística humana en su forma interior y exterior. Por lo tanto, la gramática contrastiva resultaría ser una parte de la gramática confrontativa, la parte aplicada, metodológicamente útil para la determinación de errores en el aprendizaje de una lengua. De este modo, se pretende que la diferenciación quede establecida con claridad a partir de ese momento, pero, en realidad, las respectivas designaciones no han sido siempre empleadas en sentido estricto ni por Coseriu ni por otros lingüistas y el término gramática confrontativa no ha logrado imponerse; gramática contrastiva sigue siendo el más usual (cf. Nickel 1972, 10).

1.1. Por otra parte, existe otra ciencia, la lingüística contrastiva, cuyos límites han sido confundidos con frecuencia con los de la gramática contrastiva. No es poco frecuente encontrar en los trabajos científicos las designaciones lingüística contrastiva y gramática contrastiva para el mismo objeto de estudio. En realidad, al realizar estudios contrastivos sincrónicos, no es siempre posible comparar sólo estructuras gramaticales entre sí porque a una estructura gramatical de la lengua de partida le puede corresponder un equivalente léxico o fónico en la lengua de llegada. O sea que la lingüistica contrastiva abarca los tres campos de estudio: la gramática, el léxico y la fonología (Coseriu 1970b, 17).

1.2. La comparación, según Coseriu (1970a, 177), permite constatar tanto las analogías como las diferencias de la organización lingüística y, penetrar, de este modo, en la esencia y en el funcionamiento de las lenguas para obtener valiosos datos. Por medio de ella se verifican la coherencia entre las distintas funciones que una lengua debe expresar o que normalmente expresa y, además, de una manera descriptiva, los principios de cada lengua, las relaciones entre los hechos que, considerados en forma aislada, parecen no tener sentido, pero cuya solución puede contribuir a solucionar otros. Otro cometido sería comparar las lenguas como sistemas de posibilidades de la actividad lingüística, como técnicas de la creatividad libre del ser humano, motivo por el cual Coseriu, sin olvidar nunca el concepto de energeia de W. Humboldt, propone comprobar qué es lo que se puede crear de acuerdo a cada uno de los sistemas de las lenguas comparadas.

2. Según Coseriu (1973a, 11-113; 1970b, 26-29; 1981, 189), los estudios contrastivos deben considerar los distintos niveles de estructuración lingüística de las lenguas comparadas. Esta identificación de los niveles de estructuración lingüística: el tipo, el sistema, la norma y el habla se encuentra ya entre los primeros trabajos de este destacado lingüista y

Odisea, $n^{\circ} 3,2003$ 
es básica para estudiar y comprender el funcionamiento de toda lengua en general y para los estudios contrastivos en particular. La lingüistica contrastiva debe ocuparse de los tres primeros niveles: el tipo, el sistema y la norma. La gramática confrontativa también, pero el papel más importante para ella lo desempeñan el sistema, el estudio de las funciones de una determinada lengua, y la norma. Además, Coseriu se pregunta cuánto puede o debe dejarse librado al contexto o a la situación porque para poder crear en una lengua y comprenderla es necesario conocer su sistema, pero para hablarla como los nativos hay que dominar una o varias normas.

2.1. El habla comprende los actos lingüísticos realizados en un cierto momento e incluye todo lo inédito, lo que es individual, espontáneo, incluso los errores y características particulares del hablar de un cierto individuo en un acto concreto. Siempre es necesario partir de este estrato para abstraer los otros tres niveles. La norma es repetición de modelos anteriores, la realización tradicional de las funciones, y hay que diferenciar entre una norma social, que comprende lo que es elemento común en todo el hablar de una comunidad considerada, pero sin valor funcional, y una norma individual, que abarca lo que es repetición, elemento constante en el hablar del individuo mismo. En el sistema se encuentran las oposiciones funcionales comprobables en la norma, así como también todo lo que todavía no ha sido realizado, pero que podría realizarse. El tipo lingüístico está constituido por los principios funcionales comprobados en el sistema, los tipos y las categorías de procedimientos y funciones que serían posibles, aunque en un momento histórico dado, no se comprueben. Por un lado, los distintos planos se diferencian entre sí y, por otro, se complementan y se aclaran.

3. Antes de comparar las lenguas hay que describirlas. De este hecho surge la pregunta: ¿cuál es la teoría más adecuada para realizar trabajos contrastivos? En primer lugar, la descripción de las lenguas comparadas debe llevarse a cabo con exactamente el mismo método, con la misma técnica y con el mismo aparato conceptual. Aunque diferentes teorías pueden cumplir con el mismo propósito, Coseriu (1970b, 13-15; 1981, 189) adopta el estructuralismo funcional porque éste permite el paso de los significados del habla a las funciones lingüísticas internas. Critica, sobre todo, la gramática transformacional porque ignora el eje paradigmático, es decir, las unidades funcionales que fundamentan las posibilidades de uso $\mathrm{y}$, al equiparar la estructura profunda con la designación, no distingue entre el significado y la designación. Existe una sola «estructura profunda»: la estructura semántica, la estructura del significado. La suposición de que haya una estructura profunda extralingüística no lleva a delimitar las estructuras lingüísticas, sino que, por el contrario, se renuncia a ello. No todos los investigadores concuerdan con esta opinión. Rein $(1983,18)$, en su crítica a Coseriu, considera que la gramática transformacional es un modelo descriptivo adecuado y de mayor rendimiento que otros y que ha servido para que se realizaran numerosos trabajos contrastivos.

4. Aplicamos las ideas coserianas cuando comparamos las frases infinitivas preposicionales del español y del inglés en la zona significativa causal. En este trabajo de investigación se señalan los principales aspectos que deben ser estudiados y el método 
que conviene aplicar cuando se realizan estudios de gramática contrastiva. En esta ocasión sólo daremos algunos breves datos (Mori 1980).

Si no hay estudios exhaustivos sobre un tema dado en cada una de las lenguas que se desean comparar o si el material científico existente ha sido realizado de acuerdo a teorías gramaticales diferentes, antes de hacer cualquier tipo de estudio contrastivo, es necesario delimitar el tema y comenzar por el análisis del habla, del plano del discurso; posteriormente, se identifican los hechos de norma y las fijaciones no funcionales para llegar, finalmente, al plano abstracto del sistema, de los valores funcionales, el que, a su vez, fundamentará y explicará los hechos pertenecientes a los otros dos niveles.

4.1. Es conveniente comenzar con el análisis de traducciones de las lenguas comparadas. Ya que no es factible hacer un análisis simultáneo ni bilateral ni multilateral, en un primer paso se toma una de las lenguas estudiadas como lengua de partida (L1), en este caso el español, y se buscan todos los equivalentes posibles en la lengua de llegada (L2), el inglés; en un segundo paso, el inglés será la lengua de partida o de origen (L1) y el español la lengua de llegada o meta (L2). Es éste el nivel de la equivalencia contextual, de los equivalentes en la traducción, que no coinciden uno a uno. Puede ser que entre las lenguas comparadas exista una equivalencia uno a uno, una equivalencia múltiple o bien una correspondencia cero o que los equivalentes no pertenezcan al mismo campo, por ejemplo, que una determinada construcción gramatical tenga un equivalente léxico. R. Rabadán trata los distintos enfoques del concepto de equivalencia en su libro Equivalencia y traducción: problematica de la equivalencia translémica español-ingles (69 y ss.).

4.2. Hemos comprobado que, en el plano del habla, se presenta una gran cantidad de equivalentes distintos para una determinada estructura y que no es tarea fácil justificar el motivo por el cual han sido usados ni distinguir los que son correctos de los que no lo son. Ahora bien, una estructura tiene, en principio, un valor funcional único, el que también se realiza en el habla, pero, además, otros significados de habla. A título de ejemplo, el valor funcional final de para + infinitivo se realiza también en la norma y en el habla: he comprado este libro para regalarlo, pero, a veces, la construcción española suele tener el valor de una coordinación, como en el siguiente ejemplo de García Márquez (1972, 111): ... Rebeca... se quedó paralizada de estupor y apenas pudo reaccionar para hacerle a Arcadio una señal de adiós con la mano/ ... Rebeca was paralyzed with stupor, barely able to react and wave good-bye to Arcadio, García Márquez (1971, 119).

Los equivalentes entre las dos lenguas son clasificados en tres grupos, regularidades, desviaciones justificadas y desviaciones injustificadas: a) cuando los equivalentes en las dos lenguas funcionan dentro de la misma zona significativa son regularidades; b) si una estructura de la lengua A conserva su valor funcional en el habla y tiene un equivalente en la lengua B cuyo significado de habla equivale al de la lengua A es una desviación justifica$d a$; y c) si el valor de la estructura de la lengua B no coincide con el de la lengua A es una desviación injustificada.

4.3. Durante otra etapa, al analizar el plano de la norma, se observa qué realizaciones se deben, simplemente, a algún hecho de la norma. Así, por ejemplo, en algunas regiones de 
habla española se usa ir a por agua, mientras que en otras se dice ir a buscar agua, lo que constituye una diferencia diatópica, según el subsistema empleado por el traductor.

5. En realidad, según Coseriu (1970b, 20-21; 1981, 195), siempre habría que estudiar una lengua histórica o algún fenómeno de la misma dentro de un diasistema, es decir, de la lengua seleccionada teniendo en cuenta las diferencias diatópicas, diastráticas y diafásicas - geográficas, socioculturales y estilísticas. Para efectuar la comparación hay que seleccionar diasistemas equivalentes entre las lenguas comparadas, lo que es factible cuando el principal interés es la comparación de algún aspecto de la norma de dos lenguas. Sin embargo, cuando se investiga un tema determinado con el fin de estudiar las funciones de dos o más lenguas a nivel del sistema, resulta difícil realizar un análisis tan detallado por la amplitud que cobraría el mismo. A fin de reducir esta dificultad conviene seleccionar, por ejemplo, textos en lenguaje culto, lo que no impide que la investigación abarque una mayor variedad de textos para verificar semejanzas y diferencias en la norma. También es necesario separar la «técnica libre del hablar» del «discurso repetido».

6. Otra distinción fundamental para los estudios contrastivos en la que Coseriu (1970b, 9-11) insiste con razón, es aquella entre significado funcional o significado que se presenta en la lengua y designación, ya que el significado de una determinada estructura es único en el plano del sistema, pero ésta puede tener uno o más significados contextuales y es necesario aislar lo que es funcional de lo que no lo es. Estos significados no han sido siempre diferenciados con precisión, lo que, a veces, ha llevado a que se realizaran análisis equivocados y a que no se formulara correctamente la pregunta acerca del tertium comparationis.

6.1. Ante todo, para que dos lenguas o un ámbito de las mismas pueda ser comparado, es necesario que se presuponga un tertium comparationis idéntico. Debido a la finalidad eminentemente práctica de la gramática contrastiva dirigida al aprendizaje de lenguas extranjeras, ésta se pregunta qué se dice en la lengua extranjera (lengua B) para un ámbito X de la lengua de partida (lengua A) y se estudian sólo los casos en que las lenguas no presentan analogías sino contrastes.

6.1.1. Coseriu no está de acuerdo en que el problema se plantee de este modo porque, aunque sea posible encontrar una cierta identidad ideal de los contenidos de pensamiento, no todos pueden ser separados de la realidad extralingüística. La designación es algo secundario y depende del significado de lengua y no al revés; por lo tanto es peligroso suponer que la lengua de llegada diga realmente «lo mismo» que la lengua de partida porque esto sólo se puede presuponer para los contenidos separables de una lengua. Los contenidos separables corresponden aproximadamente al significado léxico y al significado de la oración. Por ello, los equivalentes Das gefällt mir, I like this, Cela me plait y J'aime cela, Questo mi piace, Esto me gusta, Gosto disso expresan aproximadamente el mismo contenido, lo que no es válido para gefallen, to like, plaire, aimer, piacere, gustar y gostar.

6.1.2. Del mismo modo, en lo que respecta a los contenidos no separables, hay que tener en cuenta que una lengua A puede decir lo mismo que la lengua B, siempre y cuando sus 
contenidos sean más generales que los de la lengua A. Debido a ello, el contenido general del esp. negro puede precisarse con otras determinaciones con brillo, sin brillo y, de este modo, expresar los contenidos del lat. niger y ater respectivamente, pero esto no es posible al revés porque, en latín, siempre se hace referencia a la luz. También existen otros motivos por los cuales no se dice todo lo que se podría decir, tales como las fijaciones lingüísticas o las costumbres características de una comunidad. A causa de ello, Coseriu propone formular la pregunta acerca del tertium comparationis del siguiente modo: ¿qué se dice en la lengua B en una situación análoga en relación con el mismo objeto extralingüístico?, lo que, efectivamente, se hace en la práctica de la gramática contrastiva y de la traducción. Estas observaciones son de gran utilidad para los estudios contrastivos del léxico.

6.2. El tertium comparationis generalmente adoptado no tiene en cuenta que haya correspondencia cero con la segunda lengua ni que la correspondencia pueda presentarse entre distintos ámbitos de la lengua, gramatical, léxico o fónico. Por ejemplo, el francés remarquez no tiene correspondencia en alemán, a veces, Wissen Sie. La diferencia entre las estructuras tuvo un hijo y tenía un hijo se hace, en ocasiones, en alemán, por medio del léxico, bekam einen Sohn y hatte einen Sohn, pero no siempre, por ejemplo, er hatte einmal einen Sohn und hat ihn verloren - tuvo un hijo y lo perdió.

7. Coseriu (1970b, 18-19) se pregunta cómo debería ser una gramática contrastiva ideal. La gramática contrastiva no puede tener un método propio, sino que las descripciones deben preceder a la comparación para que ésta pueda efectuarse siguiendo un criterio único, por lo tanto la gramática contrastiva es un tipo de aplicación. Ella debe ocuparse primero de las funciones como tales y no de los significados de habla. Si se la considera meramente desde el punto de vista descriptivo, existe el peligro de que se equiparen categorías totalmente distintas porque coinciden en la designación o que, cuando hay acumulación de correspondientes, éstos sean tomados por casos de polisemia, por ejemplo, que tener sea un caso de polisemia por tener dos equivalentes bekommen y haben cuando, en realidad, se trata de polivalencia.

8. Coseriu (1970b, 20-30) sugiere introducir nuevos campos de estudio en la gramática contrastiva: 1. Hacer la distinción entre la determinación del hablar por medio de las cosas o por medio de la lengua. Por ejemplo, la aclaración de los nombres propios en USA está determinada por las cosas, por la cantidad de ciudades que llevan el mismo nombre: Lincoln, Nebraska; Chicago, Illinois; Madison, Wisconsin y Washington, D.C. 2. Diferenciar entre las unidades diatópicas, diastráticas y diafásicas dentro de una lengua histórica (dialectos, niveles y estilo). Así, en Francia, las diferenciaciones socioculturales son más frecuentes que en Alemania, mientras que en Alemania prevalecen, principalmente, las diferencias regionales, por ejemplo, en los chistes. 3 . También se debe tener en cuenta la diferenciación entre discurso repetido y técnica libre del hablar. Como ejemplos de lenguaje repetido se cuentan, entre otros, el empleo de refranes en español, de citas de óperas en italiano y de la Biblia en comunidades protestantes. 
8.1. En lo que respecta a las estructuras gramaticales, es necesario constatar y distinguir lo siguiente: a) los planos gramaticales, que variarán según la lengua estudiada: las unidades mínimas, la palabra, los grupos de palabras, la cláusula, la oración y, eventualmente, el texto; b) las características de estos planos: la superordenación, la subordinación, la coordinación y el reemplazo (pronominalización); c) los ámbitos de la gramática: la gramática constitucional (la morfología en sentido amplio), la gramática funcional (paradigmática funcional de los distintos planos) y la gramática relacional (relaciones entre los diferentes paradigmas, incluso las transformaciones); y d) los niveles de estructuración de la técnica del hablar (y con ello diferentes niveles de «gramaticalidad»: la norma, el sistema y el tipo (diferenciación que también es válida para el léxico y la fonética) y que ya mencionamos anteriormente.

8.2. Los planos gramaticales no son los mismos en todas la lenguas. Siguiendo a Halliday, Coseriu opina que tanto el de las unidades mínimas como el de la oración son indispensables. El tipo y la extensión de estos planos difiere en las distintas lenguas, por ejemplo, en italiano y en español, es posible superordenar piove y llueve, ya que estas formas verbales pueden corresponder al plano de la oración e incluso al del texto, lo que no ocurriría con sus equivalentes en alemán, en francés y en inglés *regnet, *pleut, *rains. Un ejemplo de subordinación es el siguiente: el grupo de palabras King of England puede funcionar como una palabra: The King of England's army, hecho que no se presenta en las lenguas romances. Entre los numerosos ejemplos de reemplazo, figura la posibilidad de que, en alemán, las formas verbales perifrásticas se puedan retomar por medio del auxiliar, por ejemplo Hast Du schon gegessen? -Habe ich, lo que no rige para el rumano.

8.3. Las diferencias gramaticales aparecen en los planos constitucional, funcional y relacional. Así, en el caso de lat. Romae, Romam, Roma; esp. en Roma, a Roma, da Roma e it. a Roma , da Roma, el latín y el español se diferencian desde el punto de vista constitucional, pero no del funcional ni del relacional. En cambio, el español y el italiano se asemejan constitucional, pero no funcionalmente. Las diferencias constitucionales se reconocen fácilmente, no así las funcionales ni las relacionales.

9. Finalmente, otro límite que Coseriu (1970c; 1981, 185 y ss.) desea establecer es aquel entre lingüística contrastiva y traducción. Una y otra vez en su extensa obra y en sus clases, explica Coseriu, con su profunda base filosófica, la distinción entre sentido, significado y designación y la importancia teórica que tiene la misma para comprender el funcionamiento de las lenguas históricas, así como también para las disciplinas de enfoque contrastivo: la lingüística estructural, la gramática y la semántica estructural y, por consiguiente, para la lingüística y la gramática contrastivas y para la traducción.

9.1. La primera distinción es aquella entre significado de lengua y significado de habla. Siguiendo a Leibniz, opina que una estructura tiene un «valor funcional» único en la lengua aunque la misma estructura pueda tener varios valores en el plano del habla. Dicha distinción trae aparejada aquella otra entre significado y designación ya comentada anteriormente (cf. 6 y ss.). 
9.2. Sólo el significado es propio de cada lengua histórica y está dado por las oposiciones funcionales de cada lengua y puede ser estructurable lingüísticamente, pero no la designación, que se relaciona con los hechos y objetos extralingüísticos. El significado es el valor lingüístico de un signo o de una construcción. La designación es la relación con lo designado (hechos y objetos), con la realidad extralingüística o esta realidad extralingüística misma. Se tiene que separar lo que es verdaderamente significado lingüístico de las restricciones de empleo no determinadas lingüísticamente sino por el conocimiento de las cosas. Por ejemplo, en español, el plural «reflexivo» puede interpretarse como reflexivo (nos lavamos, nos peinamos) o como recíproco (nos vemos, nos escribimos), pero, en ocasiones, estas formas tienen el significado contrario: nos lavamos (mutuamente); nosotras nos escribimos asi (en un cuento donde hablan las letras).

9.3. Los significados se realizan en los actos de habla con la finalidad de designar, o sea que el significado es una posibilidad determinada y no la designación en sí. Por ejemplo, la construcción con $x$ tiene un significado muy general de concomitancia, $x$ está presente, $\mathrm{y}$ una gran cantidad de posibilidades: la expresión con el cuchillo no tiene significado instrumental, sino que significa el cuchillo está presente en este hecho. Instrumental puede ser la designación, lo que se sabe no por la lengua sino por el conocimiento de las cosas. También hay otros usos con azúcar, con María, etc. En varios de sus trabajos trata Coseriu (1973b y 1973c) los diferentes tipos de significado: léxico, categorial, instrumental, estructural y óntico.

9.4. El significado y la designación han sido confundidos en estudios contrastivos de enfoque transformacional y se ha considerado que dos lenguas tienen la misma estructura profunda por coincidir en la designación, es decir, porque las dos dicen lo mismo en una situación determinada (Coseriu 1970c, 107; 1973c, 10 y ss.). Comprobamos esta observación en el trabajo de enfoque transformacionalista de T. Krzeszowski (1971), quien compara las estructuras del inglés: Seymour sliced the salami with a knife y Seymour used a knife to slice the salami con sus respectivos equivalentes en polaco y sostiene que las estructuras equivalentes tienen la misma estructura profunda aunque éstas sean diferentes en la estructura superficial.

Si adoptamos el punto de vista de Coseriu, dichas estructuras son equivalentes en la designación, pero tienen significados diferentes dentro de una misma lengua y en comparación con las de otras lenguas. Un año más tarde, Krzeszowski $(1972,77)$ cambia de opinión y rechaza la igualdad entre estructura profunda y superficial y opina que lo que tienen en común ambas oraciones es la estructura semántica aunque se diferencien en la superficie, hecho que permite a los hablantes reconocer las estructuras equivalentes y distinguirlas de aquellas que no lo son. Sin embargo, también ahora, dicha estructura semántica común a ambas lenguas no es otra cosa que la designación.

10. Una consecuencia muy provechosa de la distinción anterior para los estudios contrastivos es la existente entre correspondencia de lengua y equivalencia contextual. Según Catford (1965, 27 y 49), un equivalente contextual es un texto o la porción de un texto de la lengua de partida que, en una ocasión particular, es el equivalente contextual de un texto o de parte de un texto de la lengua de llegada. A pesar de no tener el mismo significado 
pueden ser intercambiables en una situación. Un correspondiente formal es toda categoría de la lengua de llegada (unidad, clase, estructura, elemento de estructura, etc.) que funciona, en un nivel más elevado, tan aproximadamente como sea posible, como el de la lengua de partida. Esta correspondencia sólo puede ser aproximada y la probabilidad de que se presente es mayor cuando la comparación se realiza a un nivel de abstracción más alto.

10.1. Dado que aceptamos la diferenciación de Coseriu, preferimos emplear el término correspondientes en el plano de la lengua, del significado, para las estructuras comparadas entre las cuales puede constatarse igualdad, semejanza o diferencia en los aspectos constitucional, funcional y relacional. Así, por ejemplo, en el plano constitucional, las construcciones españolas con $a$, para o por más infinitivo se asemejan a la construcción con to más infinitivo, pero difieren en la forma negativa. En español se usa para + no + infinitivo y en inglés in order not + to + base verbal. En el plano funcional, las frases españolas acusan marcadas diferencias con la frase inglesa porque cada una de ellas tiene un significado funcional único y se oponen paradigmáticamente entre sí, mientras que la frase con to + infinitivo no se opone paradigmáticamente a otras (Mori 1980, 116-121 y 186-197).

11. Según Coseriu $(1981,184)$, el sentido, que se presenta en un texto, o en una serie de actos lingüísticos relacionados, surge no sólo por el uso de la lengua sino, principalmente, por el conocimiento del marco cultural extralingüístico y, al mismo tiempo, por hechos lingüísticos que se refieren a ese marco. Hay casos, por ejemplo, en las preguntas, respuestas, órdenes, etc., en los que el sentido puede darse con medios lingüísticos, pero, por lo general, está determinado por la situación y el contexto.

12. De Hegel, adopta Coseriu (1981, 186 y ss.; 1977, 220 y ss.) la idea de que cada palabra de nuestra lengua despierta en nosotros nuestras propias imágenes de ciertos objetos y que otra lengua no sólo no tiene las mismas palabras sino que tampoco tiene las mismas imágenes. Como dos lenguas no tienen la misma concepción del mundo, las traducciones no pueden dar exactamente el mismo sentido que la versión original, pero, en realidad, no se traducen las imágenes. La traducción se ocupa de textos y de contenidos de textos. Los significados de lengua no se traducen. «Traducir» significa encontrar significados en la lengua de llegada para construir designaciones que expresen lo mismo que las designaciones de la lengua de partida. En un sentido más estricto significa «trasladar» lo expresado en los textos. De este modo, si se traduce venire del italiano al español, hay que comprobar si se trata de un movimiento en dirección a la segunda persona o en dirección hacia la primera persona, el hablante: en el primer caso se traducirá ir y en el segundo venir.

12.1. La distinción precedente es esencial y contribuye a delimitar el campo de la lingüística contrastiva del de la traducción. La lenguas son sistemas significativos - con posibilidades propias de designar, lo que quiere decir que pueden designar «lo mismo» por medio de significados diferentes. La igualdad del significado es más una excepción que un hecho normal. La tarea de la traducción es decir lo mismo con significados fundamentalmente diferentes. En una etapa, el traductor procede «semasiológicamente», de los significados de la lengua A hacia lo designado extralingüísticamente, y en otra «onomasiológicamente», 
es decir de las designaciones hacia los significados de la lengua B. La correspondencia o equivalencia es una equivalencia de las designaciones.

13. La lingüística contrastiva (1981) es una comparación sincrónica: se ocupa de las analogías y de las diferencias entre las lenguas, con más exactitud, entre los sistemas de significado. Para ello hay que considerar una de las lenguas comparadas como un sistema de designación («organización del mundo») y la otra como organización del mismo mundo por medio de otros significados y deben compararse los respectivos sistemas. Esta vez, Coseriu propone otros tipos de tertium comparationis: un sistema categorial universal (conjunto de tipos de funciones, procedimientos y paradigmas correspondientes) o un sistema de designación independiente, por ejemplo, una tercera lengua o un sistema de designación exterior construido para este fin. Ninguno de estos dos tipos de tertium comparationis es totalmente apropiado ya sea por los resultados incompletos a los que llevaría el primero y las dificultades que ofrece el segundo.

Como ya hemos visto, la lingüística contrastiva surgió como gramática contrastiva y, a veces, se sigue utilizando este término. Kühlwein y Wilss $(1981,7-8)$ rechazan la diferenciación entre lingüística contrastiva y lingüística confrontativa porque una lingüística que estudiara sólo las diferencias y no las unidades estructurales que constituyen los sistemas sería una contradicción en sí. Según Coseriu, cuando no sólo se comparan paradigmas y procedimientos gramaticales sino funciones y diferencias funcionales, pronto se nota que la gramática contrastiva no puede ser sólo gramática en sentido estricto sino que tiene que considerar otros sectores al mismo tiempo porque los contenidos semejantes o idénticos no son estructurados de la misma forma en las lenguas comparadas.

13.1. Sin embargo, una lingüística contrastiva a nivel del sistema o del tipo lingüístico no es muy provechosa para la traducción porque, en la traducción, no se trata de correspondencias de significado sino de variantes de los significados, de clases de designación de una lengua en comparación con las de otra y las variantes de significado contienen más rasgos que las unidades funcionales correspondientes porque incluyen los rasgos constitucionales y situacionales. A causa de esto, una lingüística contrastiva corresponde a la norma, es decir al nivel del cual se ocupa la traducción ya que la traducción no sólo se relaciona con el texto sino también con el hablar y debería determinar las clases de equivalentes. Coseriu ha señalado que, ya en otras oportunidades, se han establecido las variantes de una lengua en comparación con las de otras, sobre todo en el campo del léxico.

13.2. Otros aspectos que deben ser considerados son los siguientes: a) los significados ocupan el primer lugar — son posibilidades abiertas de la designación y no al revés, un ejemplo entre otros: en italiano se dice buon senso, en español sentido común y en alemán gesunder Menschenverstand, lo que no quiere decir que buono, común y gesund signifiquen lo mismo; b) Los diferentes tipos de fijaciones, sobre todo las situacionales y las materiales, por ejemplo, ciertos términos de una lengua se usan en una determinada situación con mayor o menor frecuencia que sus equivalentes en otra. Hay equivalencia entre esp. enterarse, regatear, estrenar, despedirse y los términos italianos apprendere, mercanteggiare, inaugurare, prendere congedo, pero los términos italianos se emplean 
menos que los españoles. Además, existen fijaciones materiales, por ejemplo, fr. noir et blanc y esp. blanco y negro.

13.3. Una lingüística contrastiva completa que se ocupara del nivel de la norma y analizara el uso de los significados exhaustivamente daría todos los equivalentes y, de este modo, se asemejaría a una traductología de las dos lenguas. Para este tipo de lingüística contrastiva sería la traducción una fuente constante y sus resultados serían una gramática de la traducción o un diccionario de la traducción. Coseriu $(1981,194)$ expresa el deseo y la necesidad de llevar a cabo tal tipo de estudios contrastivos que no han sido realizados hasta ahora. Sin embargo, una lingüística contrastiva ideal no puede proveer todo para la traducción porque hay límites bien marcados entre ambas: lo que se traduce son textos y ellos contienen, también, lenguaje repetido. Este campo del discurso repetido no ha sido estudiado todavía adecuadamente desde el punto de vista contrastivo. La traducción debe confrontarse con el hecho de que, en un texto, se dan distintas lenguas funcionales simultáneamente, mientras que una lingüística contrastiva coherente sólo puede ser hecha para sistemas lingüísticos únicos, para «lenguas funcionales».

Generalmente, la lingüística contrastiva se ocupa de equivalentes existentes y en la traducción aparecen nuevas designaciones para las cuales hay que crear otras designaciones. Los textos tienen su propia tradición, la que no coincide con la tradición de una lengua. La traducción abarca no sólo la designación sino el sentido y designaciones semejantes pueden expresar algo distinto. El límite más marcado entre la lingüística contrastiva y la traducción es el «conocimiento de las cosas».

14. De lo dicho se deduce que Coseriu, repetidas veces, se propuso delimitar teóricamente el objeto de cada una de las ciencias contrastivas y, basándose en principios teóricos y ejemplos prácticos, nos muestra cuáles son los caminos transitables para llegar a realizar estudios más exhaustivos en campos todavía no estudiados. Expresamos el deseo de que las distinciones teóricas mencionadas sean tenidas en cuenta al realizarse investigaciones contrastivas y que sus propuestas para que se amplíe y complete el campo de los estudios contrastivos se conviertan en una realidad.

\section{BIBLIOGRAFÍA}

Catford, J. C. 1965. A Linguistic Theory of Translation. An Essay in Applied Linguistics. Londres: 1965.

Coseriu, E. 1970a. «Zusammenfassung der Ergebnisse». Probleme der kontrastiven Grammatik. Jahrbuch 1969 des Instituts für Deutsche Sprache in Mannheim, H. Moser, ed., vol. VIII. Düsseldorf: Schwann: 175-177.

- 1970b. «Über Leistung und Grenzen der kontrastiven Grammatik». Probleme der kontrastiven Grammatik. Jahrbuch 1969 des Instituts für Deutsche Sprache in Mannheim, H. Moser, ed., vol. VIII. Düsseldorf: Schwann: 9-30.

-1970c. «Bedeutung und Bezeichnung im Lichte der strukturellen Semantik». P. Hartmann y H. Vernay eds. Sprachwissenschaft und Übersetzen. Munich: Hueber: 104-121. 
-1973a. «Sistema, norma y habla». Teoría del lenguaje y lingüistica general. Madrid: Gredos, 3a. ed.: 11- 113.

-1973b. «Semantik und Grammatik». Neue Grammatiktheorien und ihre Anwendung auf das heutige Deutsch, Jahrbuch 1971 des Instituts für Deutsche Sprache in Mannheim, vol. XX. Düsseldorf: Schwann: 77-89.

-1973c. «Die Lage in der Linguistik». Innsbrucker Beiträge zur Sprachwissenschaft, W. MeID, ed., Conferencia 9. Innsbruck.

-1977. «Lo erróneo y lo acertado en la teoría de la traducción». El hombre y su lenguaje. Estudios de teoría y metodología lingüística. Madrid: Gredos: 214-239.

—1981. «Kontrastive Linguistik und Übersetzung: ihr Verhältnis zueinander». Kontrastive Linguistik und Übersetzungswissenschaft. Akten des Internationalen Kolloquiums Trier/Saarbrücken 25.-30.9.1978, W. KüHLweIn, G. Thome y W. WiLsS eds., Munich: Wilhelm Verlag: 183-199.

Di Pietro, R. J. 1972. «Kurze orientierende Bemerkungen zur Untersuchung sprachlicher Verschiedenheit». Reader zur kontrastiven Linguistik, G. Nickel, ed., Fráncfort del Meno: Athenäum: 136-146.

García Márquez, G. 1972. Cien años de soledad, 29a. ed. Buenos Aires: Editorial Sudamericana.

-1971. One Hundred Years of Solitude, trad. por G. Rabassa. Nueva York: Avon Books.

KRZESZOWSKI, T. 1971. «Equivalence, congruence and deep structure». NiCKEL, G., ed., Papers in Contrastive Linguistics. Cambridge: 1971: 37-48.

—1972. «Kontrastive Generative Grammatik». G. NiCKEL, ed., Reader zur kontrastiven Linguistik. Fráncfort del Meno: Athenäum: 39-58.

KüHLWEIN, W. y WILSS, W. 1981. «Kontrastive Linguistik und Übersetzungswissenschaft». Kontrastive Linguistik und Übersetzungswissenschaft. Akten des Internationalen Kolloquiums Trier/Saarbrücken 25-30.9.1978, KüHLweIn, W., Thомe, G. y WiLss, W. eds., Munich: W. Fink: 7- 17.

Mori, O. 1980. Frases infinitivas preposicionales en la zona significativa causal. Estudio contrastivo español-inglés. Tubinga: Gunter Narr.

Nickel, G. 1972. Zum heutigen Stand der kontrastiven Sprachwissenschaft. Reader zur kontrastiven Linguistik, G. NicKEL, ed., Fráncfort del Meno: Athenäum: 7-14.

RABADÁN, R. 1991. Equivalencia y traducción: problemática de la equivalencia translémica inglés-español. León: Universidad, Secretariado de Publicaciones.

REIN, K. 1983. Einführung in die Kontrastive Linguistik, Darmstadt: Wissenschaftliche Buchgesellschaft.

ZaBrocki, L. 1970. «Grundfragen der konfrontativen Grammatik». Probleme der kontrastiven Grammatik, Jahrbuch 1969 des Instituts für Deutsche Sprache in Mannheim, H. Moser, ed., vol. VIII. Düsseldorf: Schwann: 31-52. 